\title{
Calculation of Level Density Parameter of Nuclear Reaction Using Neural Network
}

\author{
Rizal Kurniadi ${ }^{1)^{*}}$, Yuda Satya Perkasa ${ }^{1)}$, Abdul Waris ${ }^{1)}$ and Suwoto ${ }^{2)}$ \\ 1) Nuclear Physics \& Biophysics Research Division, \\ Faculty of Mathematics and Natural Sciences, \\ Institut Teknologi Bandung, Indonesia \\ ${ }^{2)}$ Center for Reactor Technology and Nuclear Safety \\ National Nuclear Energy Agency of Indonesia \\ *e-mail: rijalk@fi.itb.ac.id
}

\begin{abstract}
Level density parameter (LDP) calculation is dependent upon shell correction value, which usually is obtained by using Strutinsky method. The Strutinsky method is method that uses energy levels from certain potential as main input. Therefore for each mass number it strongly needs the energy levels calculation. The energy levels computation is time-consuming process; hence, the new application of computation technique is needed to reduce computation time. This paper explains the application of neural network in LDP calculation. Based on knowledge from box type and harmonic potentials, LDP is predicted by using arbitrary potential
\end{abstract}

Keywords: LDP, Neural Network, Strutinsky method, Shell correction, Harmonic potential

\section{Introduction}

Level density plays an important role in calculating the nuclear reaction model statistically, such as in calculating the evaporation model of nuclear reaction, spallations reaction measurements, and in studies of intermediate-energy of heavy ion collision $^{1)}$. Although there are some theoretical approaches that have been developed to study the level density ${ }^{2-4)}$, one of the parameters that holds very important role in the level density calculation is the level density parameter (LDP) ${ }^{5}$. The commonly used of level density parameter is the energy dependent value $^{6,7)}$.

The asymptotic value of the LDP is reached at the infinite excitation energy ${ }^{8)}$. With this approach, the variation of its LDP value is small. This is caused by the highly excitation state. This approach is different from the shell correction approach, which gives bigger value of the variation of the LDP. Shell correction is resulted from the difference between nuclear mass experiment and semi empirical nuclear mass ${ }^{9}$.

In level density study, semi empirical nuclear mass is influenced mainly by pair and shell correction. In shell correction, fission barrier determines the variation value of eigen energy to smooth curve parts. To simplify the complexity of the problem, the simple potential interaction such as infinite square well or harmonic oscillator is usually chosen and the GaussHermite folding technique is used ${ }^{10)}$.

The effect of simplifying the complexity results in the limitation of the potential interaction, which is in contradiction with the real problems in nuclear reaction model. In nuclear reaction model, the more complicated the potential interaction, the more accurate the nuclear data will be resulted. Based on this hypothesis, this paper will discuss the advantages of using the supervised neural net to calculate LDP. The supervised neural net that is chosen is the back propagation one. This method is described in details in ${ }^{11)}$.

Basically, back propagation requires a number of vector inputs and vector target. Through these vectors, the neural network will learn to identify the patterns given and predict other patterns having similar basic pattern as the ones given. The pattern during the LDP calculation is composed of the configuration potential parameters, atomic mass, and atomic number, while the vector target is determined by its shell correction value. Hence, the obtained neural network knowledge is the knowledge to recognize the relationship among the potential parameters, atomic mass, and atomic number against the shell correction value. The training method discussed in this paper is the conjugate gradient algorithm $^{12)}$, while neural architecture used contains 100 neurons in hidden layer and one neuron in output layer.

The potential parameters used as the attribute for the input vectors are the difference between the area below the curve of the box potential and the area below the curve of the harmonic potential. Its potential variation is determined by the difference below these two curves. One of interaction potential that will be obtained from these parameters is the wood-saxon potential type $^{13)}$.

\section{Formulation of Method}

The value of level density is approximated by the th following formula ${ }^{14)}$,

$$
a(A, Z, U)=\tilde{a}(A)\left[1+\frac{\delta E}{U}\left(1-e^{-\gamma U}\right)\right]
$$

Parameter $\tilde{a}$ is asymptotic value at infinite excitation energy $U$. There are three-semi empirical formulas for $\tilde{a}^{14)}$. In this paper, the Ignatyuk formula represents the asymptotic parameter has been employed. 


$$
\tilde{a}=0.154 A+6.3 \times 10^{-5} A^{2}
$$

$U$ is the value excitation energy that was approximated by the following equation (3) ${ }^{15)}$.

$$
U=a_{c} t_{c}
$$

and $t_{C}$ is the critical temperature

$$
\begin{aligned}
& t_{c}=0.567 \Delta \\
& \Delta=\frac{12}{\sqrt{A}}
\end{aligned}
$$

As for the semi empirical level density parameter, $a_{C}$, its calculation has been proposed by GilbertCameroon $^{2)}$, which can be written as follows.

$$
a_{c}=\frac{\pi^{2}}{6} g\left(e_{f}\right)
$$

With $g\left(e_{f}\right)$ as a single particle level density at fermi level of $e_{f}=33 \mathrm{MeV}, g\left(e_{f}\right)$ is calculated by formula ${ }^{16)}$

$$
g\left(e_{f}\right)=\frac{2 \sqrt{2}}{\pi \hbar^{2}} \int \sqrt{e_{f}-V(r)} r^{2} d r
$$

where $V(r)$ in equation (5) is oscillator harmonic potential and infinite box potential. Both potentials are used to introduce the knowledge of neural network, which will be used

$$
V(r)= \begin{cases}\gamma \omega r^{2}+V_{0} & \text { oscillator harmonic for all } r \\ V_{0} & \text { box potential for } r \leq R_{\text {nucl }} \\ \infty & \text { box potential for } r>R_{\text {nucl }}\end{cases}
$$

Because the interaction is not the interaction of of non-mean field approximation, coulomb interaction used is the interaction of point to sphere technique ${ }^{17)}$

$$
V_{\text {coul }}(r)=\frac{Z_{1} Z_{2}}{R_{c}} f_{c}(x)
$$

Where

$$
\begin{aligned}
& f_{c}(x)=\frac{1}{x} \tanh \left(\frac{3}{2} x+\frac{5}{8} x^{3}\right) \\
& R_{c}=R_{1}+R_{2} \\
& x=\frac{r}{R_{c}}
\end{aligned}
$$

then the interaction depicted by $V(r)=V_{\text {noncoul }}(r)+V_{\text {coul }}(r)$, which replace the conventional one $V\left(\vec{r}_{1}\right)=\int V\left(\vec{r}_{12}\right) d^{3} \vec{r}_{12}$.

The iteration technique is applied to equation (1) to get $\mathrm{LDP}^{18)}$

$$
\begin{aligned}
& a_{0}=\tilde{a}(1+\delta E) \\
& U^{(n)}=a_{n} t_{c}^{t} \\
& a_{n+1}=\tilde{a}\left[1+\frac{\delta E}{U^{(n)}}\left(1-e^{-\gamma U^{(n)}}\right)\right]
\end{aligned}
$$

$\delta E$ parameter in equation (9) is called shell correction, which is calculated using the formula,

$$
\delta E=M_{\text {exp }}-M_{\text {calc }}
$$

Nuclear masses experiment $M_{\text {exp }}$ are taken from AME2003 table $^{19)}$ while $M_{\text {calc }}$ is calculated using binding energy formula

$$
\begin{aligned}
& B(A, Z)=A_{v}\left(1-k_{v} I^{2}\right) A-a_{s}\left(1-k_{s} I^{2}\right) A^{2 / 3} \\
& -\frac{3}{5} \frac{e^{2} Z^{2}}{R_{0}}+E_{\text {pair }}-E_{\text {shell }}-a_{k} A^{1 / 3} \\
& -a_{0}-f_{p} \frac{Z^{2}}{A}-W|I|
\end{aligned}
$$

in which all coefficients at the equation (11) were compiled by Royer ${ }^{20)}$

$$
E_{\text {pair }}= \begin{cases}-\frac{11}{\sqrt{A}} & Z, N \text { odd } \\ 0 & \text { Aodd } \\ \frac{11}{\sqrt{A}} & Z, N \text { even }\end{cases}
$$

Where equation (12) and Eshell represent the pair and shell correction at binding energy formula, which is determined $b^{21)}$

$$
E_{\text {shell }}=\int_{0}^{n}(\varepsilon(n)-\bar{\varepsilon}(n)) d n
$$

$\varepsilon_{n}$ is an eigen energy while $\bar{\varepsilon}(n)$ is smooth curve that it was calculated from ${ }^{22,23)}$

$$
\begin{aligned}
& \bar{n}(\varepsilon)=\int_{-\infty}^{\infty} \bar{g}\left(\varepsilon^{\prime}\right) d \varepsilon^{\prime} \\
& \int_{0}^{N} \bar{\varepsilon}(n) d n=\int_{-\infty}^{\bar{\lambda}} \varepsilon \bar{g}(\varepsilon) d \varepsilon \\
& \int_{0}^{N} \bar{\varepsilon}(n) d n=\frac{\bar{\lambda}^{4}}{8(\hbar \omega)^{3}}-\frac{\bar{\lambda}^{2}}{16 \hbar \omega} \\
& \int_{0}^{N} \bar{\varepsilon}(n) d n=\frac{\pi}{10} \frac{\overline{\lambda^{5 / 2}}}{E_{o}^{3 / 2}}-\frac{3 \pi}{16 E_{o}} \bar{\lambda}^{2}+\frac{1}{4 E_{o}^{1 / 2}} \bar{\lambda}^{3 / 2}
\end{aligned}
$$

The value of fermi level $\bar{\lambda}$ in the equation was obtained from the relation

$$
n(\bar{\lambda})=N
$$

The vector used as the input vector is constructed from $\alpha, \beta, A$ and $Z$. $\alpha$ is the difference between the area constructed by box potential curve and the area of harmonic potential curve below zero potential value.

While $\beta$ is the difference between the area constructed by the two potentials above zero value. $A$ and $Z$ are atomic mass and atomic number respectively. The target vector is composed of values that it is obtained from the conventional calculation 
procedure. The training data are taken from the representative of each value $A$, while testing data are taken from isotope in the training data. The training algorithm that was implemented was CGA combined with the updating formulation.

$$
\begin{aligned}
& p_{k}=-g_{k}+\beta_{k} P_{k-1} \\
& \beta_{k}=\frac{\Delta g_{k-1}^{T} g_{k}}{g_{k-1}^{T} g_{k-1}}
\end{aligned}
$$
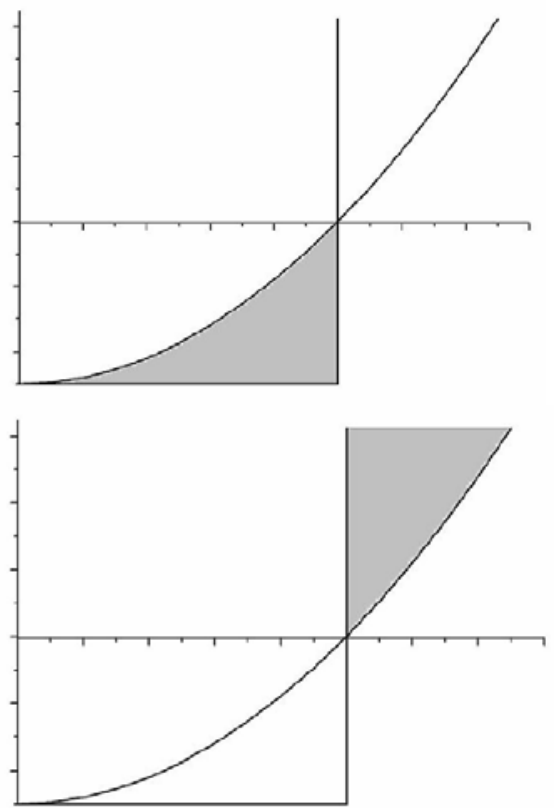

Figure 1. the $\alpha$ area (top) and the $\beta$ area (bottom)

\section{Results and Discussion}

The testing result on the two potentials is displayed in Figure 2
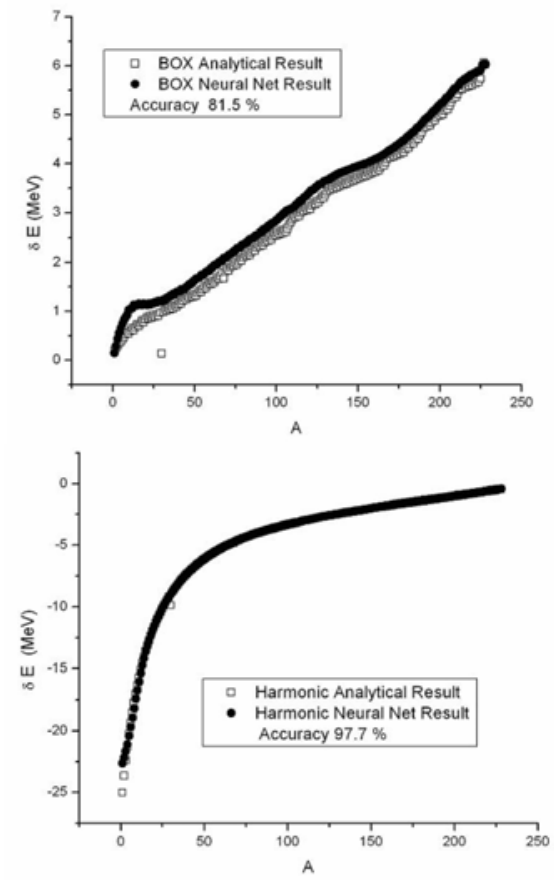

Figure 2. Accuracy curves from testing process
Figure 2 shows that the accuracy of both trainings is above $70 \%$. The accuracy for Box Potential reaches $87 \%$ and harmonic potential has the accuracy of $97 \%$. The data indicate that the reliability of the neural network knowledge is significant. The number of Epoch for both the Box Potential and Harmonic Potential is 60000. In addition, the learning rate of training used is 0.1 . The performance curves are showed by figure 3 .

Based on the knowledge acquired, the prediction for the shell correction value from both potentials is made. Figure 4 depicts this predicted shell correction

Figure 4 shows that $\delta E$ box potential is higher than harmonic potential; hence, the predicting value $\delta E$ has the possibility to exist within the range of the two potentials.
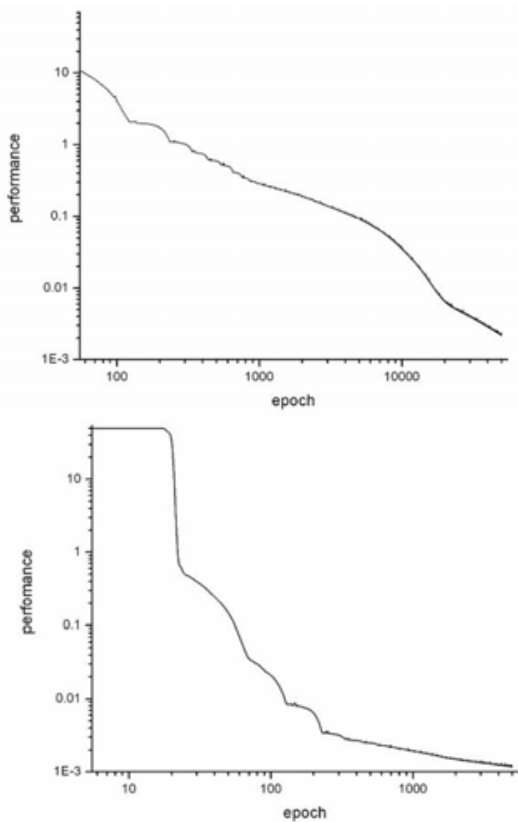

Figure 3. Training performance curves from box potential (top) and hrmonic potential (bottom)

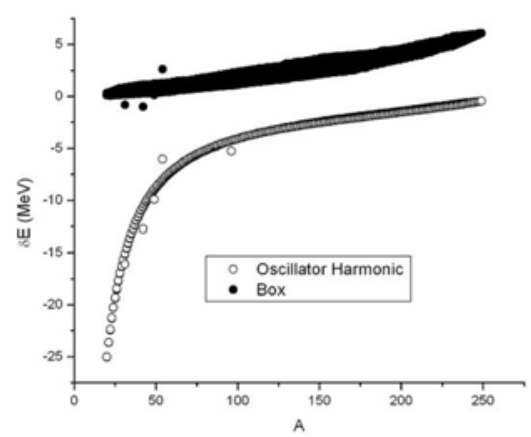

Figure 4. LDP predicted result from both neural knowledge

If the value $\alpha$ for predicting is zero, the negative value for its potential is a box shape. Normally, the predicting value should be higher than the value produced by harmonic potential. Considering that the knowledge acquired is from both the Box Potential system and the Harmonic Potential 
system, knowledge for predicting is obtained by means of the formula of averaged knowledge.

$$
\langle\overrightarrow{N e t}\rangle=\frac{1}{2} \sum_{i=1}^{2} \overrightarrow{N e t_{i}}
$$

Because the value of shell correction is varied, which fluctuates according to its oscillating pattern, $\beta$ value for the vector input was approximated by equation(19)

$$
\beta=\beta_{0} \sum_{l=0} P_{l}\left(\sin \left(\beta_{0}\right)\right) \exp \left(-\beta_{0}\right)
$$

Where $P_{l}$ is legendre polynomial and $\beta_{0}$ is $\beta$ value from the training data. Based on this approximation, the predicted results $\delta E$ obtained are shown in figure 5 ,

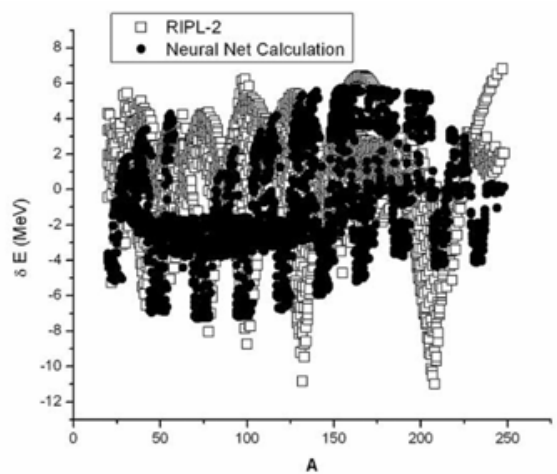

Figure 5. Neural net (filled circle) and LDP shell correction result (unfilled box)

By using ignatyuk formula and RIPL-2 iterative procedure, LDP for RIPL-2 and neural network method is calculated. The calculation results are illustrated in figure 6.
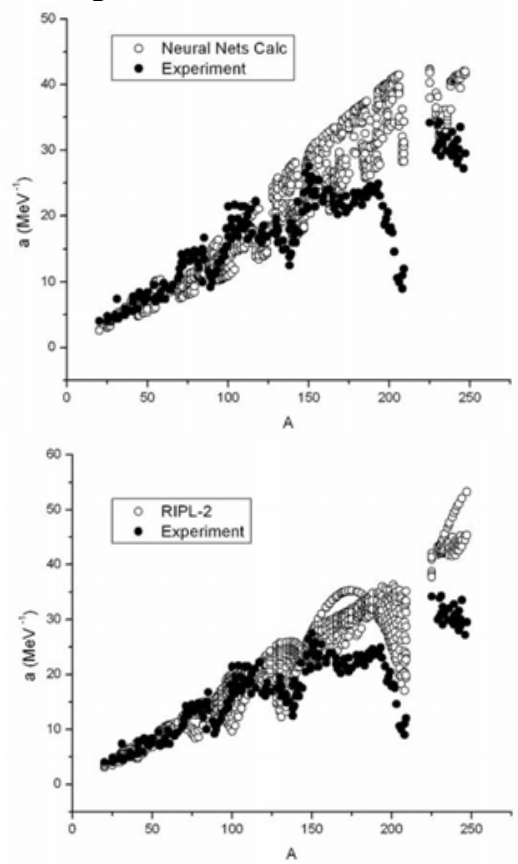

Figure 6. The LDP neural network result (top) and RIPL-2 calculation (bottom)
Figure 6 shows clearly that RIPL-2 calculation deviates at about $\mathrm{A}=170$, likewise neural network, in which the result has significant discrepancy at $\mathrm{A}=$ 170. Although neural network has a significant discrepancy at $A=170$, around the area of mass 225, the result of neural network is closer to the experiment compared with the value of RIPL-2.

\section{Conclusions}

This work have performed a new calculation procedures of LDP, with an emphasis on techniques that permit to overstepped the eigen value problem solving. The calculation proceeds according to four steps :

(a). Calculation of box and harmonic potential knowledge;

(b). Carrying out the accuracies of knowledge;

(c). Determination of $\alpha$ and $\beta$ functions for input vectors;

\section{(d). Predicting process;}

There are still some answered questions, and more work needs to be done before a final assessment can be made, but tentatively it appears that the overstepping of the eigen value problem in LDP calculation here does provide two advantages :

(a). Reducing computation time and;

(b). Building arbitrary potential data base that suitable in LDP calculation;

\section{Acknowledgements}

This research is fully funded by ITB research grant (riset KK ITB) 2008

\section{References}

1. K. der Merr, et al., Spallation Yields of Neutrons Produced in Thick Lead/bismuth Targets by Protons at Incident Energies of 420 and $590 \mathrm{MeV}$, Nucl Inst. and Meth. In Phys. Res. B., 217, 202. 2004.

2. A. Gilbert, A. G. W. Cameron, A Composite Nuclear-level Density Formula with Shell Corrections, Can. J. of Phys., 43, 1446, 1965

3. S. Hilaire, Level Densities, Workshop on Nuclear Data and Nuclear Reactors : Physics, Design and Safety, 2000.

4. An Yu, Hu Jimin, Trial Densities for the Extended Thomas-Fermi Model, Nucl. Phys. A., 598, 1, 1995.

5. M. Ksczmarczyk, The Empirical Description of the Level Density Parameter $a$ in the Semiclassical Model, J. Phys. G : Nucl. Part. Phys., 26, 253, 2000.

6. P. Demetriou, and S. Goriely, Microscopic Nuclear Level Densities for Practical Applications, Nucl. Phys. A., 695, 95, 2001.

7. S. Hilaire, Energy Dependence of the Level Density Parameter, Phys. Lett. B., 583, 264, 2004. 
8. D. Bucurescu, and T. von Egidy, Systematics of Nuclear Level Density Parameters, J. of Phys. G : Nucl. Part. Phys., 31, S1675, 2005.

9. P. Moller, at al., Nuclear Ground-State Masses and Deformations, at Data and Nucl. Data. Tables 59, 185, 1995.

10. B. Nerlo-Pomorska, K. Pomorski and J. Bartel, Shell Energy and the Level-density Parameter of Hot Nuclei, Phys. Rev. C., 74, 34327, 2006.

11. D. E. Rumelhart, G. E. Hinton, and R. J. Williams, Learning Internal Representations by Error Propagation, Parallel Data Processing Vol. 1, Chap 8, The MIT Press, Cambrige, MA, 318, 1986.

12. R. Flecther and C. M. Reeves, Function Minimization by Conjugate Gradients, Computer Journal, 7, 149, 1964.

13. R.D. Woods and D.S. Saxon, Diffuse Surface Optical Model for Nucleon-Nuclei Scattering, Phys. Rev., 95, 577, 1954.

14. Handbook for Calculations of Nuclear Reaction data, RIPL-2, IAEA-TECDOC 1506, 2006.

15. M. Rizea, et al., Calculation of Nuclear Level Density Relevant for Thorium-based Reactors, Rom. Rep. in Phys, 57, 757, 2005.
16. J. N. De, S. Shlomo, and S. K. Samaddar, Level Density Parameter in a Refined Thomas-Fermi Theory, Phys. Rev. C., 57, 1398, 1998.

17. R. Anni, Regularized Legendre Series of Improved Nearside-farside Decomposition for Charged Particle Scattering, Phys. Rev. C., 67, 057601, 2003.

18. M. Herman et al., EMPIRE Nuclear Reaction Model Code Ver 2.19 Lodi, 2005.

19. G. Audi, A. H. Wapstra, and C. Thibault, The AME 2003 Atomic Mass Evaluation: (II). Tables, Graphs and References, Nucl. Phys. A., 729, 337, 2003

20. R. Royer, and C. Gautier, Coefficients and Terms of the Liquid Drop Model and Mass Formula, Phys. Rev. C, 73, 067302, 2006.

21. V. M. Strutinsky, Shell Effects in Nuclear Masses and Deformation Energies, Yad. Fis. 3, 614, 1996.

22. M. Bolsterli et al., New Calculation of Fission Barriers for Heavy and Superheavy Nuclei, Phys. Rev C., 5, 1050, 1972.

23. B. Mohammed-Azizi and D. E. Medjadi, Connection Between the Strutinsky Level Density and the Semiclassical Level Density, Phys. Rev. C., 74, 054302, 2006. 